\title{
Intelligent Classifier for E-Nose Systems
}

\author{
Dechen Pelki ${ }^{1}$, Javier Bajo ${ }^{1}$, and Sigeru Omatu ${ }^{2}$ \\ ${ }^{1}$ Department of Artificial Intelligence, Technical University of Madrid, Madrid, Spain \\ ${ }^{2}$ Osaka Institute of Technology, Osaka, Japan \\ pelki.dechen.pelki@alumnos.upm.es, jbajo@fi.upm.es, \\ omatu@rsh.oit.ac.jp
}

\begin{abstract}
This paper briefly discusses the state-of-the-art of e-noses and classifiers used in analyzing the response data from E-Nose systems and presents an idea about how to face off this kind of problems using ensembles of classifiers.
\end{abstract}

Keywords: E-Nose system, ensemble of classifiers.

\section{Introduction}

An E-Nose is an instrument used for the automated detection and classification of odors, vapors and gases, thus mimicking the human olfactory apparatus [3]. It is used in the perfumes, food, beverages and biomedical industries to classify various complex odors. A general E-Nose system consists of a sample handling, a detection and a data processing system. After considering the samples and methods specifications for the sample handling, the detection system consists of an array of sensors and then the pattern recognition techniques for analyzing the response data generated by the detectors [5]. In this paper, we revise the state-of-the-art about e-noses and the classifiers used in the existing e-noses. Then we obtain some preliminary conclusions and propose an ensemble of classifiers as an innovative technique for an e-nose system developed in Japan.

\section{Related Work}

Most of the applications for e-noses concentrate on four major areas; food, medical diagnosis, environmental monitoring and bio-process control [7]. Rodriguez et al. [6] used an A-Nose to discriminate Colombian coffee into simple and complex odors using PCA and MLP BPNN with LOO cross validation method. A portable e-nose Pen2 identified the quality grade of green tea by extracting feature vectors using PCA and validating using LDA and BPNN [10]. It is possible to directly classify the sensor data using BPNN only; using a TGS 800 series Smart E-Nose for controlling coffee quality [8] but it leads to overfitting. It is also possible to extract features to reduce data dimension using PCA only without validating or using any supervised classifiers such as the work by Berna et al. [2], in comparing 2 e-noses for detecting changes in tomato aroma profiles of two different cultivars and a quartz crystal microbalance based e-nose for detection of bacterial contaminated milk [1]. However, Dutta et al. 
[4] combined 3D-scatter plot, Fuzzy C Means, SOM for extracting features, and used MLP, PNN and RBF for classifying the 6 eye bacteria data. This proposal enhanced the performance of Cyranose 320 e-nose, but it can be very difficult to implement and incorporates a high degree of complexity. Chen et al. [3] employed a MDC with a combination of KNN, LDA and PNN, which provided an increased overall classification accuracy compared to the use of any single individual classifier.

\section{Conclusions and Research Goal}

E-Noses mostly use feature extraction methods followed by a pattern recognition method. The typical feature extraction linear methods used are PCA, LDA and ICA [9]. The task of a classifier is to use the feature vector provided by the feature extractor to assign the object it represents to a category [7]. Currently, the existing E-Nose systems employ a feature extraction method followed by a single classifier for analyzing the response data except for two cases where the feature extraction methods have been combined and an appropriate classifier that performs specifically better with the combination has been suggested in [3][4]. The methods proposed are also specific to the kind of odor that is being detected. Our aim is to propose an ensemble of classifiers to analyze the response data, trying to take advantage of the combined responses of classifiers and focusing on a particular e-nose system created at the OIT in Japan. More specifically, we propose the use of MLP and SVM to explore the combination of different classifiers in an effective manner.

\section{References}

1. Ali, Z., O'Hare, W.T., Theaker, B.J.: Detection of Bacterial Contaminated Milk by means of a Quartz crystal Microbalance Based Electronic Nose. Journal of Thermal Analysis and Calorimetry 71, 155-161 (2003)

2. Berna, A.Z., Lammertyn, J., Saevels, S., Natale, C.D., Nicolai, B.M.: Electronic nose systems to study shelf life and cultivar effect on tomato aroma profile. Sensors and Actuators B 97, 324-333 (2004)

3. Chen, H., Goubran, R.A., Mussivand, T.: Improving the Classification Accuracy in Electronic Noses Using Multi-Dimensional Combining (MDC). IEEE (2004)

4. Dutta, R., Hines, E.L., Gardner, J.W., Boilot, P.: Bacteria classification using Cyranose 320 electronic nose. BioMedical Engineering Online (2002)

5. Peris, M., Escuder-Gilabert, L.: A 21st century technique for food control: Electronic noses. Elsevier (2009)

6. Rodriguez, J., Duran, C., Reyes, A.: Electronic Nose for Quality Control of Colombian Coffee through the Detection of Defects in "Cup Tests". Sensors (2010)

7. Scott, S.M., James, D., Ali, Z.: Data Analysis for electronic nose systems. Springer (2006)

8. Shilbayeh, N.F., Iskandarani, M.Z.: Quality Control of Coffee Using an Electronic Nose System. American Journal of Applied Sciences 1(2), 129-135 (2004)

9. Stone, J.V.: Independent component analysis: an introduction. Trends Cogn. Sci. 6, 59 (2002)

10. Yu, H., Wang, J., Xiao, H., Liu, M.: Quality grade identification of green tea using the eigenvalues of PCA based on the E-nose signals. Sensors and Actuators B 140, 378-382 (2009) 